\title{
Identifying the Type of Face To Face Connection from STEP AP203 File
}

\author{
Monir M. Koura ${ }^{1} \&$ AsmaaHamed ${ }^{2}$ \\ ${ }^{1}$ Design \& Production Dept., Faculty of Eng., Ain Shams University, Cairo, Egypt \\ ${ }^{2}$ Engineering Design and Production Technology, Faculty of Engineering and Material Science, German \\ University in Cairo, Cairo, Egypt
}

\begin{abstract}
Feature recognition has been always an important link between CAD and CAM. Feature recognition is applied in successive stages. Firstly, feature entities extraction should be applied, followed by an algorithm of how to relate the entities together. Finally, the feature recognition algorithm is applied. In this paper, a logic for identifying face-face interaction is applied. The type of face-face interaction is needed for further use in feature recognition. A methodology is introduced to determine the relation between faces. The relation iseither concave, convex or non-joined faces. The connected edge between the faces is determined, and the included angle is calculated to determine the relation between them. The algorithm has been implemented using java programming language. Case studies have been applied for prismatic parts and successfully identified face-face relation as illustrated and shown for different faces combinations.
\end{abstract}

Keywords: Step AP203;Feature Extraction;Face recognition; Faces Interaction Types; Java programming.

\section{Literature Review}

Several researches related to automatic feature recognition are presented in this section focusing on how each technique extraction process is done.

JaiderOussama et al.[1] presented a system for recognizing isolated and interacting features for rotational parts. The system is organized into three main modules; the first module extracts and stores the data from a STEP AP203 file, the second module recognizes isolated features according to a rule based technique and in the last module a feature generator system was developed to recognize interacting features through the analysis of adjacent surfaces of each feature. This later module determines whether the feature is perfect or imperfect. The system built gives several suggested combinations of the parts which are generated by the features generation module.

Haiyan Li et al. [2] presented a hint based reusable feature shape recognition system. The reusable feature shape recognition system is used to extract the geometric entities. An explanation to the generic shape was presented. The generic shapes were classified into vertex class, edge class and face class. The generic shapes also could be isolated or hybrid. The isolated generic shapes are the shapes that are independent of the other shapes. The hybrid generic shapes are the shapes composed of different isolated shapes. The hybrid features are classified into containing, fusing and intersecting hybrid features.

Abouel Nasr et al. [3] proposed a methodology for automatic feature recognition of simple and complex features. The methodology is based on an object oriented structure and the input is a STEP AP203 file. A rule based techniques was used to recognize the features based on a geometric reasoning. The developed methodology was implemented and verified through a case study. The proposed methodology recognized only limited features. Also, it ignores the feature interaction between the features to be recognized.

Sunil et al. [4] developed a hybrid technique for automatic feature recognition of prismatic parts. A graph and rule based hybrid techniques are used to recognize interacting features. The output of the system is a text file with a native feature based format similar to STEP. For each feature recognized post-processing routine were written to compute the feature parameters. The developed techniques didn't deal with protrusions and complex volumetric interactions which are essential in manufacturing processes.

Tan et al. [5]introduced a methodology of recognition of blind and through holes. The system used STEP file as an input to the system. The feature recognition system consists of three modules. The first module generates the STEP file. Module number two extracts the geometric information from the STEP file. Module number three is the feature recognition module using rule based technique. The study is limited only for feature recognition of holes without any feature interaction.

Rameshbabu and Shunmugam [6] developed a hybrid approach using volume subtraction and face adjacency graph to recognize prismatic parts. A weak link was found between feature recognition and setup planning. A setup planning system was developed for generating a setup sequence of machining. The proposed setup planning system consists of feature recognition module and setup planning module.The hybrid feature recognition method used both volume subtraction and face adjacency graph. Feature volume is obtained by 
subtraction of the $3 \mathrm{D}$ model of the components from the raw material. The feature was represented by the feature volume faces.

Sivakumar and Dhanalakshmi [7] presented a methodology for extraction of manufacturing features from STEP file for cylindrical parts. The algorithm was based on rule based technique and was implemented on several types of features. A program was developed to generate the $\mathrm{NC}$ codes from the extracted data of the part model. A comparison has been made between the data extracted from STEP file and the actual measured data using CMM. The feature recognition methodology has been limited only for simple cylindrical features with no feature interaction. prosmatic parts.

Koura O.M. [8] presented a complete model for the extraction of features from STEP CAD files for

Sreeramulu and Rao [9] developed an automatic feature recognition system for rotational parts. The developed methodology consisted of several algorithms such as data exchange algorithm from the STEP file, two turning feature recognition algorithms for identifying primary features, algorithm for recognition of holes and an algorithm for recognition of special turning features.

Kumar et al. (10)introduced a CAPP system with feature based modeling and process parameters selection for prismatic micro parts. The input to the system is STEP XML file. The STEP XML file is used for feature extraction and interactive feature recognition. Then, knowledge based process parameters selection is used for the given feature information.

It is clear that the urge for a system that identifies the concavity and convexity of edges is needed. The aim of this research is to determine an effective logical methodology to extract the face-face interactions and identify its type.

\section{Calculation Of Normal Vectors}

The proposed methodology for the identification of the type of the connected edge is shown in Fig.1 .The faces could be connected concavely, convexly or there is no common edge between them. The angle $\theta$ is needed to be calculated in order to determine whether the faces connection are concave or convex.

$$
\cos \theta=\frac{\overrightarrow{\text { normal of vector } 1} . \overline{\text { normal of vector } 2}}{\mid \text { normal of vector } 1|| \text { normal of vector } 2 \mid}
$$

If the angle $\theta$ between them is between zero and less that $180^{\circ}$ the face-face connection is convex, and if it is more than $180^{\circ}$ and less than $360^{\circ}$ the face-face connection is concave. If no common edge is determined between the faces, these faces are non-connected faces.

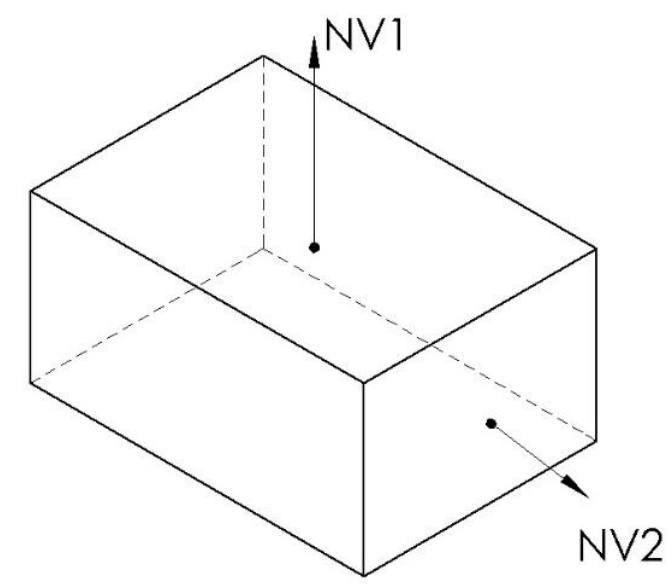

Fig 1 Orientation of Normal Vectors

The normal vector for each face is calculated. The following equations are used to calculate the normal vector.

$$
\begin{aligned}
& \vec{N}=\overrightarrow{A B}+\overrightarrow{A C} \\
& \overrightarrow{A B}=\left(x_{B}-x_{A}\right) \vec{\imath}+\left(y_{B}-y_{A}\right) \vec{\jmath}+\left(z_{B}-z_{A}\right) \vec{k} \\
& \overrightarrow{A C}=\left(x_{C}-x_{A}\right) \vec{\imath}+\left(y_{C}-y_{A}\right) \vec{\jmath}+\left(z_{C}-z_{A}\right) \vec{k}
\end{aligned}
$$

The three points $\mathrm{A}, \mathrm{B}$ and $\mathrm{C}$ are random points from a face to determine the normal vector to such a face. 


\section{Face Type Identification}

The normal vector for each face has 2 possible directions, each in each side of the face. It is needed which one of the two normal vectors will be used for the face-face interaction. It is essential to determine first the type of the face in order to decide which normal vector will be used. Fig 2 represents an overview of the normal vector re-orientation.

Depression and protrusion identifying is used to re-orient the normal vector. A depressed face is a face that all its points are equal in $\mathrm{Z}$ and is less than the $\mathrm{Z}$ maximum of the part. A protruded face is a face that all its points are equal in $\mathrm{Z}$ and is equal to the $\mathrm{Z}$ maximum of the part as shown in Fig 3. Re-orientation of normal vectors of depressed faces is shown in Fig 4. Re-orientation of normal vectors of protruded faces is shown in Fig 5.

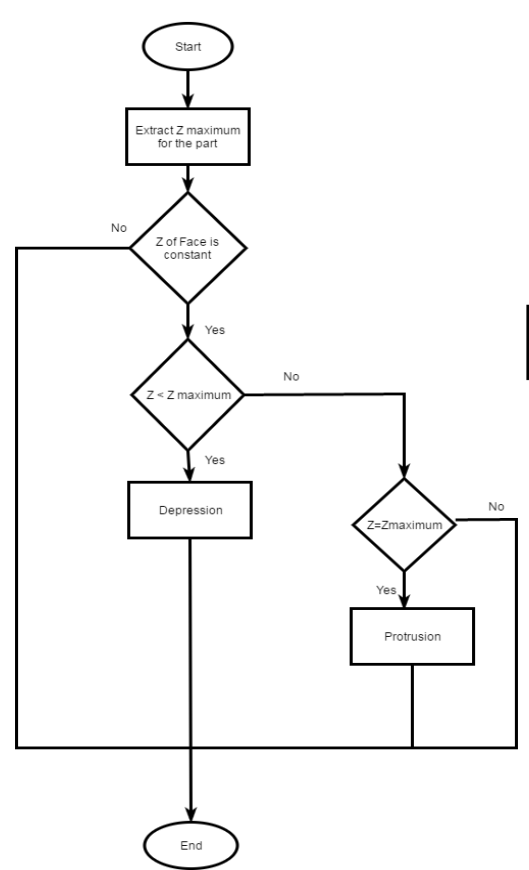

Fig 2 Overview Normal Vector Re-orientation

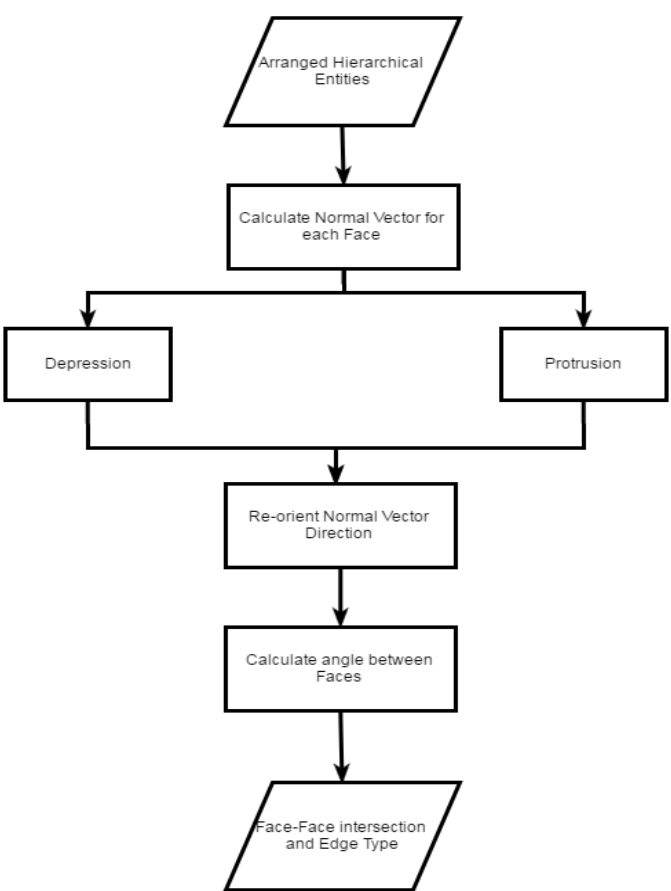

Fig 3 Depression vs. Protrusion

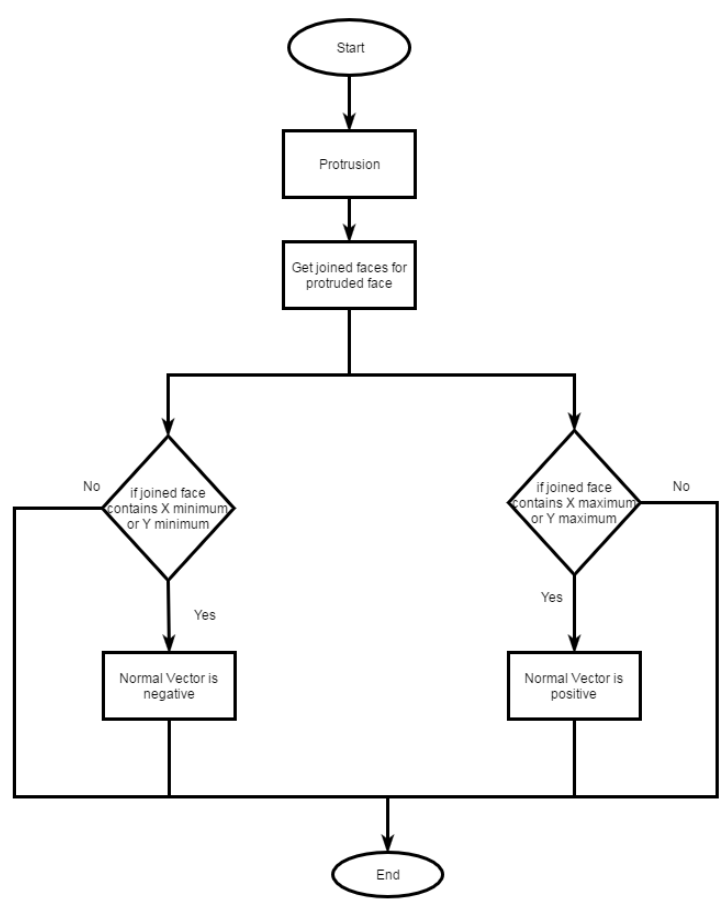

Fig 4 Normal Vector Re-orientation For Depressed faces

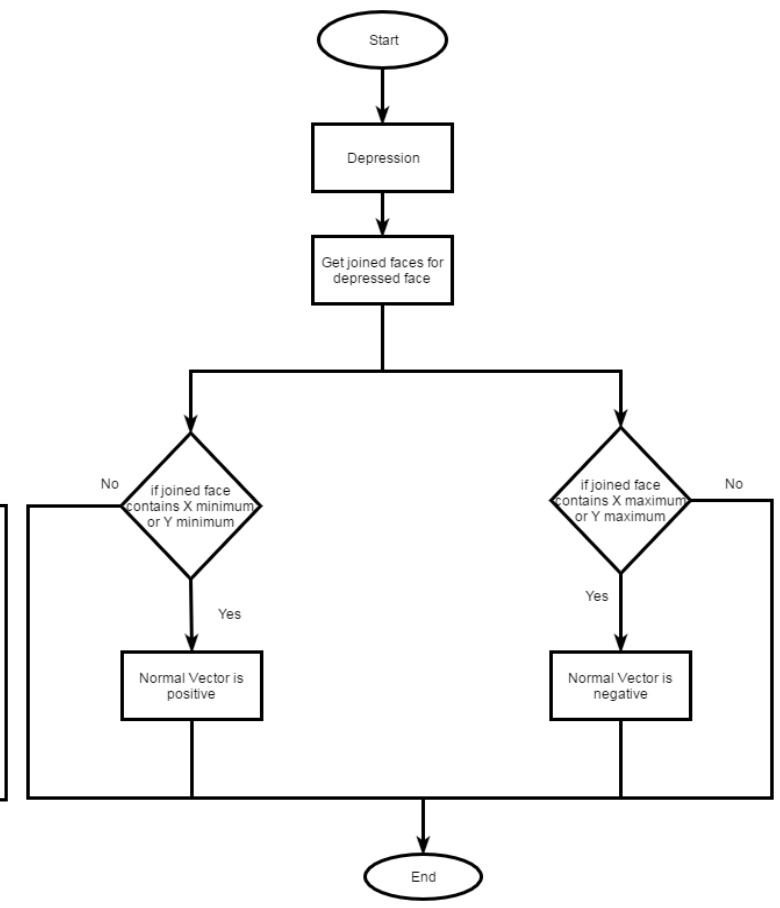

Fig 5 Normal Vector Re-orientation for Protruded faces 


\section{Case study}

A case study has been implemented to verify the proposed methodology. The 3-D model was created using Solidworks 2014 as shown in Fig. 6 .The part consists of 11 faces. The geometric information was extracted from the STEP AP203 file. There were 55 face-face interaction extracted by the implementation and their types were identified according to the proposed methodology. The face-face interactions were successfully identified and listed below in Table 1.

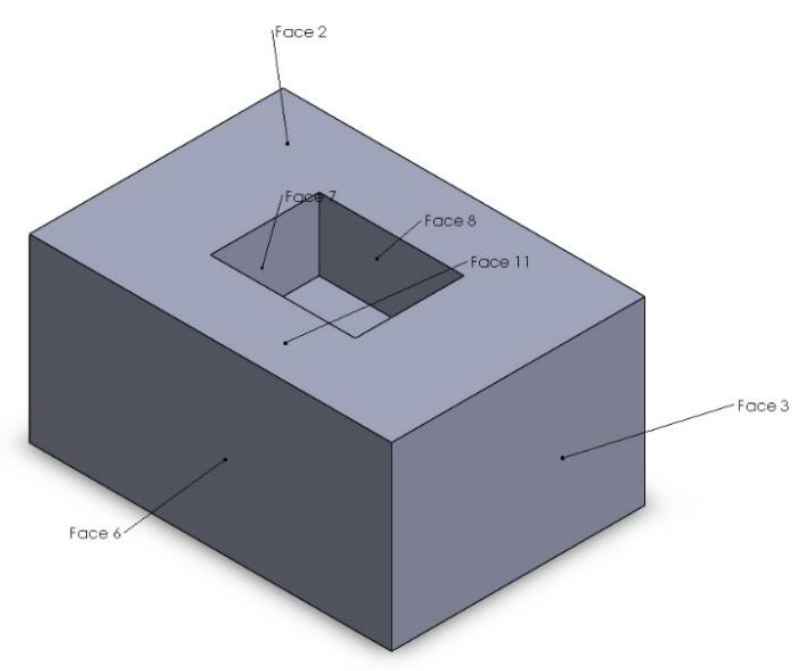

Fig 6 Case Study 1

Table 1 output of the face-face connection

\begin{tabular}{|c|c|}
\hline Face 1-Face 2 & convex \\
\hline Face 1-Face 3 & no common Edge \\
\hline Face 1-Face 4 & convex \\
\hline Face 1-Face 5 & convex \\
\hline Face 1-Face 6 & convex \\
\hline Face 1-Face 7 & no common Edge \\
\hline Face 1-Face 8 & no common Edge \\
\hline Face 1-Face 9 & no common Edge \\
\hline Face 1-Face 10 & no common Edge \\
\hline Face 1-Face 11 & no common Edge \\
\hline Face 2-Face 3 & convex \\
\hline Face 2-Face 4 & no common Edge \\
\hline Face 2-Face 5 & convex \\
\hline Face 2-Face 6 & convex \\
\hline Face 2-Face 7 & no common Edge \\
\hline Face 2-Face 8 & no common Edge \\
\hline Face 2-Face 9 & no common Edge \\
\hline Face 2-Face 10 & no common Edge \\
\hline Face 2-Face 11 & no common Edge \\
\hline Face 3-Face 4 & convex \\
\hline Face 3-Face 5 & convex \\
\hline Face 3-Face 6 & convex \\
\hline Face 3-Face 7 & no common Edge \\
\hline Face 3-Face 8 & no common Edge \\
\hline Face 3-Face 9 & no common Edge \\
\hline Face 3-Face 10 & no common Edge \\
\hline Face 3-Face 11 & no common Edge \\
\hline Face 4-Face 5 & convex \\
\hline Face 4-Face 6 & convex \\
\hline Face 4-Face 7 & no common Edge \\
\hline Face 4-Face 8 & no common Edge \\
\hline Face 4-Face 9 & no common Edge \\
\hline Face 4-Face 10 & no common Edge \\
\hline Face 4-Face 11 & no common Edge \\
\hline Face 5-Face 6 & no common Edge \\
\hline Face 5-Face 7 & no common Edge \\
\hline Face 5-Face 8 & no common Edge \\
\hline Face 5-Face 9 & no common Edge \\
\hline
\end{tabular}


Identifying The Type Of Face To Face Connection From STEP AP203 File

\begin{tabular}{|c|c|}
\hline Face 5-Face 10 & no common Edge \\
\hline Face 5-Face 11 & no common Edge \\
\hline Face 6-Face 7 & no common Edge \\
\hline Face 6-Face 8 & no common Edge \\
\hline Face 6-Face 9 & no common Edge \\
\hline Face 6-Face 10 & no common Edge \\
\hline Face 6-Face 11 & no common Edge \\
\hline Face 7-Face 8 & concave \\
\hline Face 7 -Face 9 & no common Edge \\
\hline Face 7-Face 10 & concave \\
\hline Face 7-Face 11 & concave \\
\hline Face 8 -Face 9 & concave \\
\hline Face 8 -Face 10 & no common Edge \\
\hline Face 8 -Face 11 & concave \\
\hline Face 9-Face 10 & concave \\
\hline Face 9-Face 11 & concave \\
\hline Face 10 -Face 11 & concave \\
\hline
\end{tabular}

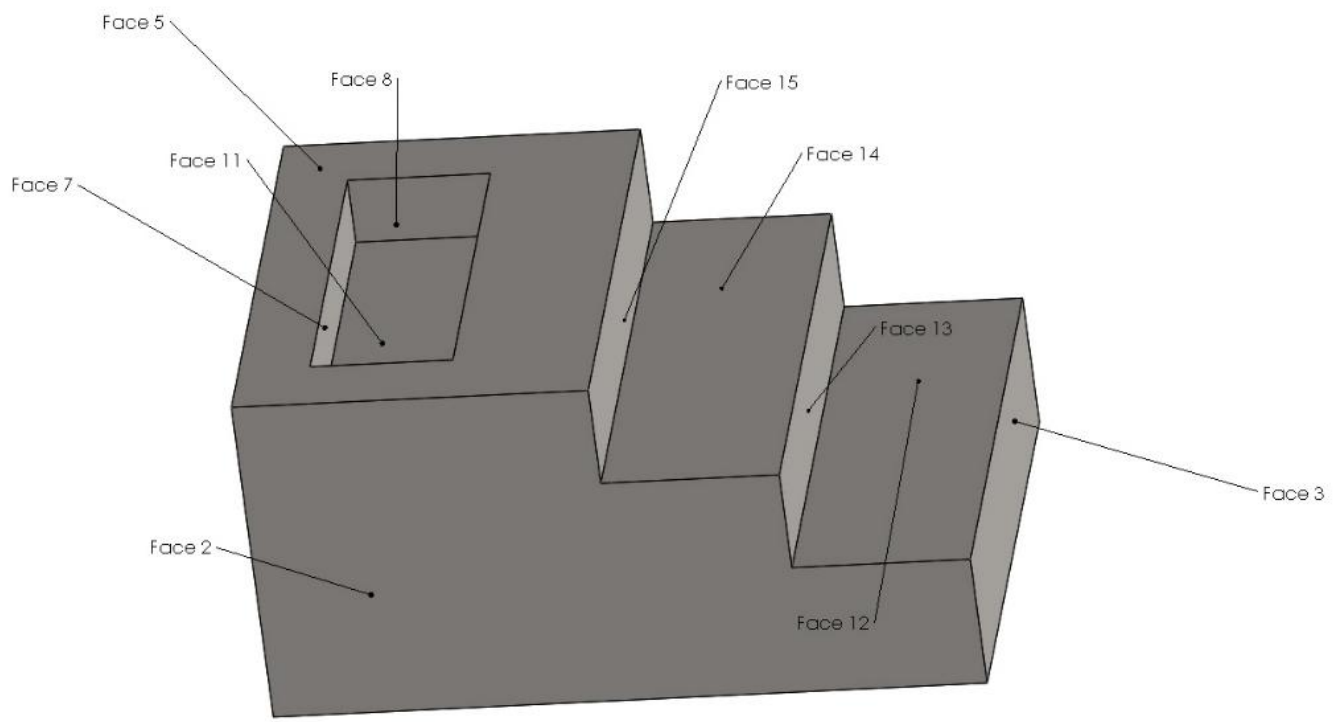

Fig 7 Case Study 2

Table 2 Part of the output of the face-face connection

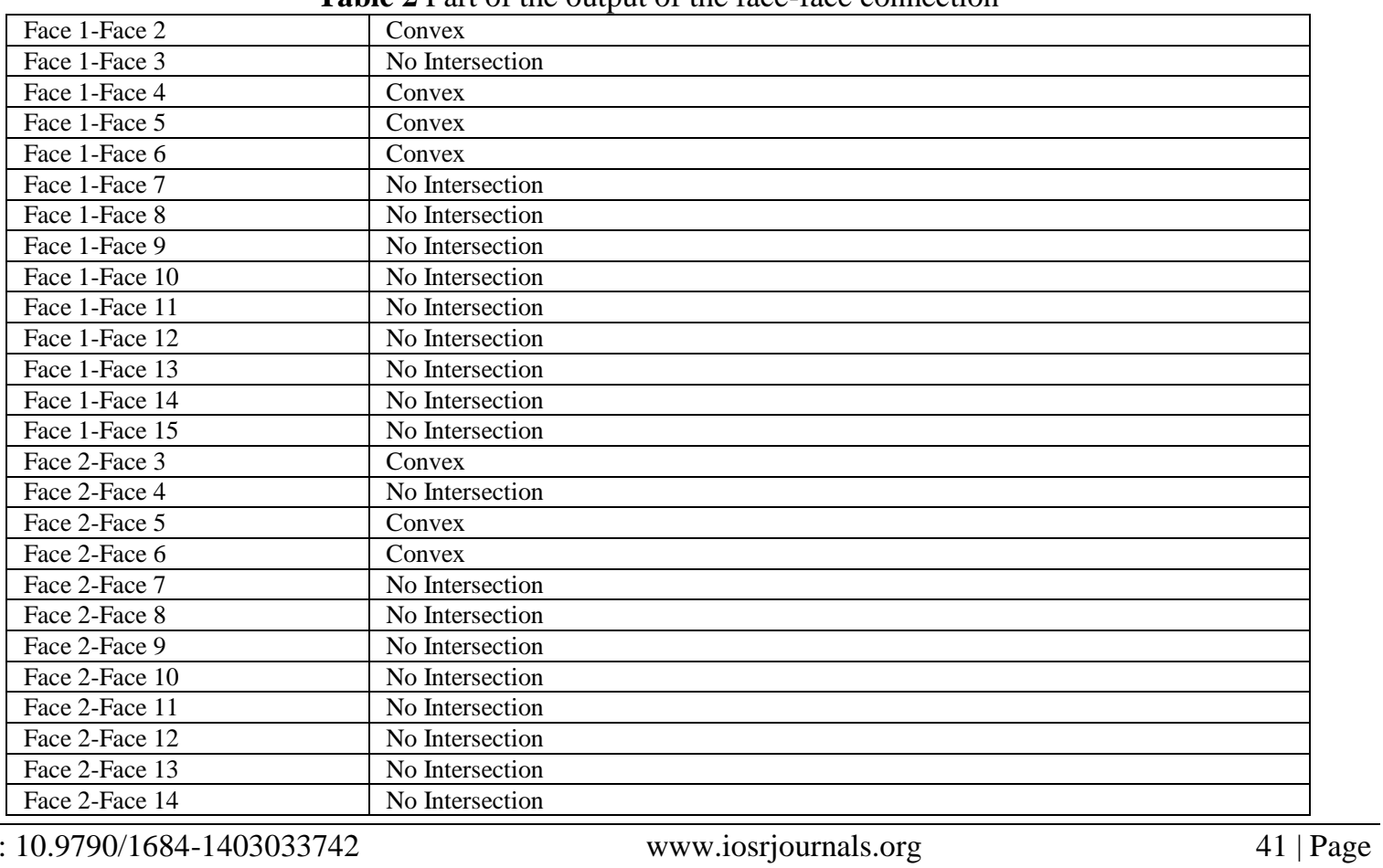




\begin{tabular}{|l|l|}
\hline Face 2-Face 15 & No Intersection \\
\hline Face 3-Face 4 & Convex \\
\hline Face 3-Face 5 & No Intersection \\
\hline Face 3-Face 6 & Convex \\
\hline Face 3-Face 7 & No Intersection \\
\hline Face 3-Face 8 & No Intersection \\
\hline Face 3-Face 9 & No Intersection \\
\hline Face 3-Face 10 & No Intersection \\
\hline Face 3-Face 11 & No Intersection \\
\hline Face 3-Face 12 & Convex \\
\hline Face 3-Face 13 & No Intersection \\
\hline Face 3-Face 14 & No Intersection \\
\hline Face 3-Face 15 & No Intersection \\
\hline
\end{tabular}

\section{Conclusions}

In the current work, a feature extraction methodology has been presented and implemented using a STEP AP203 file as an input to the system. An objected oriented algorithm has been developed using java programming language.

The presented methodology is the corner stone for an automatic feature recognition process which acts as a link between CAD and CAM. The methodology was able to determine the type of relation between faces and various edge types. Further work will be applied to recognize isolated and interacting features

\section{Acknowledgment}

The Authors wishes to acknowledge Dr. Omar M. Koura for his guidance and contribution in developing the feature extraction model from the STEP- Files

\section{References}

[1]. Manufacturing Computer Aided Process Planning For Rotational Parts. Part 1: Automatic Feature Recognition From STEP AP203 Ed2. Oussama, Jaider, Abdelilah, Elmesbahi and Ahmed, Rechia . 5, s.1. : Journal of Engineering Research and Applications, 2014, Journal of Engineering Research and Applications, Vol. 4, pp. 14-25.

[2]. Hint-based generic shape feature recognition from three-dimensional B-rep models. Li, Haiyan, et al. 2015, Advances in Mechanical Engineering.

[3]. A Feature Recognition System using Geometric Reasoining. Abouel Nasr, E., et al. s.1. : Procedia CIRP, 2014, Vol. 18, pp. 238243.

[4]. An approach to recognize interacting features from B-Rep CAD models of prismatic machined parts using a hybrid (graph and rule based) technique. Sunil, V., Agarwal, Rupal and Pande, S. s.1. : Computers in Industr, 2010, Vol. 61, pp. 686-701.

[5]. Design of a Feature Recognition System for CAD/CAM Integration. Tan, Chee, Kher, V. and Ismail, N. 8, s.1. : World Applied Sciences Journal, Vol. 21, pp. 1162-1166.

[6]. Hybrid feature recognition method for setup planning from STEP AP203. Rameshbabu, V. and Shunmugam, M. s.l. : Robotics and Computer-Integrated Manufacturing, 2009, Vol. 25, pp. 393-408.

[7]. A feature-based system for CAD/CAM integration through STEP file for sylindrical parts. Sivakumar, S. and Dhanalakshmi, V. 2013, Indian Journal of Engineering \& Materials Sciences, Vol. 20, pp. 21-26.

[8]. Focusing on the extracted data from STEP CAD files for prismatic surfaces. Omar Monir Koura, submitted to JERA in 3/12/2016, accepted for publication in 2/5/2017

[9]. A new methodology for recognizing features in rotational parts using STEP data exchange standard. Sreeramulu, D. and Rao, C. 2011, International Journal of Engineering, Science and Technology, pp. 102-115.

[10]. Feature-based ,pdelling and process parameters selection in a CAPP system for prismatic micro parts. Kumar, S.P. Leo, Jerald, J. and Kumanan, S. 10, s.1. : International Journal of Computer Integrated Manufacturing, 2015, Vol. 28, pp. 1046-1062. 